ANNALS OF

CLINICAL

NEUROPHYSIOLOGY

\title{
Transient global amnesia associated with multiple lesions in the corpus callosum and hippocampus
}

Jin-Ah Kim, Young Gi Min, and Dae Lim Koo

Department of Neurology, Seoul National University Boramae Medical Center, Seoul National University College

of Medicine, Seoul, Korea

Received: December 8, 2018

Revised: February 22, 2019

Accepted: March 9, 2019

\section{Correspondence to}

\section{Dae Lim Koo}

Department of Neurology, Seoul National University Boramae Medical Center, Seoul National University College of Medicine, 20 Boramae-ro 5-gil, Dongjak-gu, Seoul 07061, Korea

Tel: +82-2-870-2473

Fax: +82-2-831-0714

E-mail:koodaelim@gmail.com

\section{ORCID}

\section{Jin-Ah Kim}

https://orcid.org/0000-0001-9378-346X Young Gi Min

https://orcid.org/0000-0002-8091-7585 Dae Lim Koo

https://orcid.org/0000-0001-6858-6093
Transient global amnesia is a syndrome of temporary loss of short-term memory and is not accompanied by any other neurological deficit. Diffusion-weighted imaging is useful to improve the diagnostic accuracy of transient global amnesia. We report a 68 -year-old woman with multiple lesions on diffusion-weighted imaging in the right corpus callosum and left hippocampus. To the best of our knowledge, this is the first case of a diffusion-weighted imaging lesion in the body portion of the corpus callosum.

Key words: Transient global amnesia; Stroke; Magnetic resonance imaging

Transient global amnesia (TGA) is a syndrome characterized by an acute and temporary loss of anterograde and recent retrograde memory that lasts for less than 24 hours and is not associated with any other neurological deficit. ${ }^{1}$ Diffusion-weighted imaging (DWI) can reveal one or more punctate lesions in the hippocampus in patients with TGA, and is useful to improve the diagnostic accuracy of TGA. ${ }^{2}$ Here we report a patient with multiple DWI lesions in the right corpus callosum and left hippocampus. To the best of our knowledge, this is the first case of a DWI lesion in the body portion of the corpus callosum.

\section{CASE}

A 68-year-old woman without any vascular risk factors visited our emergency room with her husband, who described that she asked repetitive questions despite repeatedly being given correct answers. On September 16, 2017, she went to her bedroom at 8:30 p.m as usual, and three hours later she woke her husband up and repeatedly asked questions such as "what is the date today?" and "whose phone is this?". Her memory loss with repetitive questioning was sustained for approximately 4 hours. She had suffered from emotion- 
al stress due to family problems before the symptom onset, and experienced headache during the amnesia. She had a history of migraine, glaucoma, and a ureteric stone. No abnormal findings were observed in neurological examinations and laboratory tests. Her score on the Mini Mental State Examination (MMSE) at five hours after the symptom onset was estimated at 29, with full recovery from the transient memory loss. Brain magnetic resonance imaging (MRI) was performed four hours after the symptom onset. DWI with $a b$ value of 1,000 revealed two diffusion restrictive lesions: one in the left hippocampus and the other in the body portion of the right corpus callosum (Fig. 1A, B). Magnetic resonance angiography showed mild narrowing of the left proximal internal carotid artery. Follow-up DWI performed three days later revealed that the initial lesions were more prominent, but there were no new lesions. Serial electroencephalography recordings showed no epileptiform discharges. Holter monitoring and transthoracic and transesophageal echocardiograms revealed no evidence of a cardiogenic source of emboli. Eight months later she revisited our emergency room with a complaint of memory loss with repetitive questioning. She also experienced headache like during the first episode. She could not sleep at all because of emotional stress related to conflict with her neighborhood. After 8 hours of memory loss she recovered to a normal state, and no neurological deficit remained. Her MMSE score was 29. DWI with a $b$ value of 3,000 revealed two focal hyperintense lesions in the left hippocampus (Fig. 1C, D) in different loca- tions from the previous lesions. The previous abnormal lesions in the left hippocampus and right corpus callosum had disappeared in both DWI and T2-weighted fluid-attenuated inversion recovery imaging.

\section{DISCUSSION}

Hyperintense lesions in the corpus callosum on DWI have rarely been reported in patients with TGA. Ay et al. ${ }^{3}$ reported a TGA patient with unilateral lesions in the hippocampus and retrosplenium on DWI. Another case presented with a hyperintense lesion in the retrosplenium of the corpus callosum. ${ }^{4}$ These are the only two reported cases of retrosplenial lesions in TGA patients. It is well known that the retrosplenial cortex provides an alternative route for reciprocal connections between the anterior thalamus and the medial temporal structures, which are components of the Papez circuit. ${ }^{5}$ However, our patient presented with a lesion in the corpus callosum (and specifically in the body portion), rather than in the retrosplenium. The corpus callosum is anatomically located between the fornix and the cingulate gyrus, which are the main components of the Papez circuit. The corpus callosum and fornix are nearly in contact at the posterior part of their body portions. Based on the anatomical relationship between the corpus callosum and the Papez circuit, we postulated the possible effect of the corpus callosum on the memory process. In our case, the lesions in the corpus
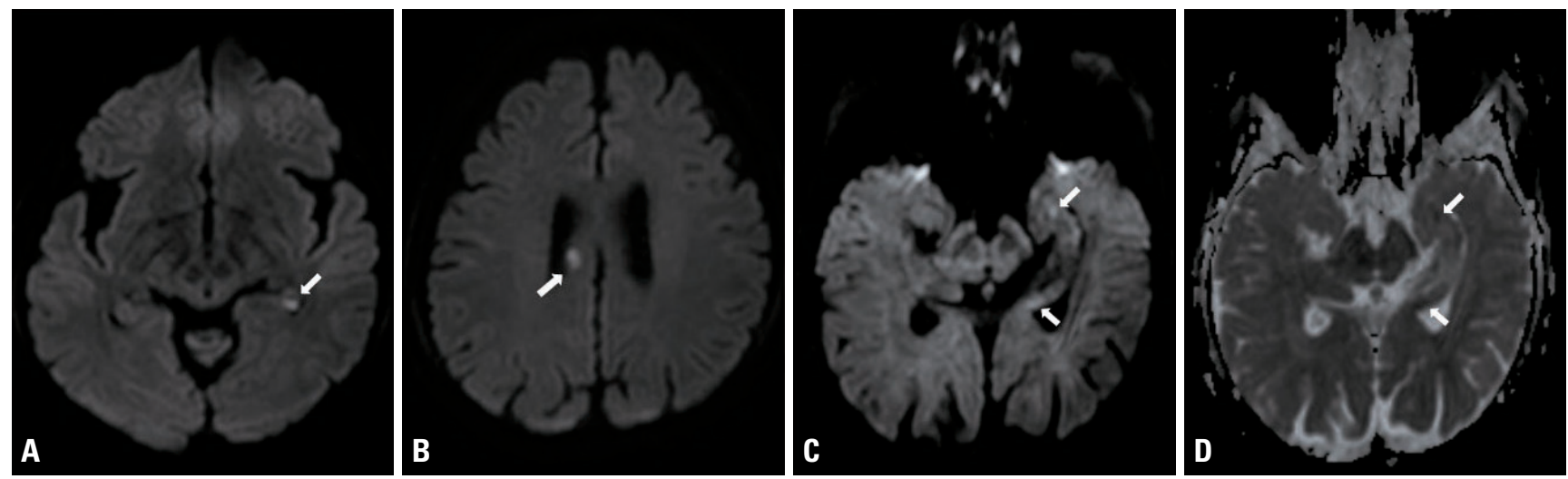

Fig. 1. Hyperintensities on a magnetic resonance imaging scan in a patient with recurrent transient global amnesia (TGA) episodes. In the first TGA episode, diffusion-weighted imaging (DWI) revealed hyperintense lesions in ( $A$, arrow) the left hippocampus and ( $B$, arrow) the right corpus callosum. In the second TGA episode, (C, arrows) hyperintense lesions on DWI and ( $D$, arrows) apparent-diffusion-coefficient-matched low signal intensities were located in the left hippocampus. 
callosum and right hippocampus could have affected the amnestic symptom. Further studies with larger samples should attempt to elucidate the role of the corpus callosum in this memory circuit.

The pathophysiology of TGA remains unclear, although several pathophysiological mechanisms have been proposed: arterial ischemia, migrainous phenomenon, cortical spreading depression, venous congestion, and epilepsy. ${ }^{6}$ In our patient, MRI revealed multiple diffusion restrictions in the hippocampus and corpus callosum over two episodes. Even though hyperintense lesions on DWI usually reflect acute ischemia, there remained controversy about the etiology of TGA in our patient. There was precipitating emotional stress in both episodes, and she had a history of long-standing migraine and suffered from headache during both episodes. She had no vascular risk factors for atherosclerosis and no embolic source for stroke. Furthermore, the locations of the two lesions-one in the hippocampus and the other in the corpus callosum-are not compatible with the cerebrovascular territory. In addition, the locations of these lesions did not match small-vessel occlusion or other neurological conditions. Cortical spreading depression or epileptic seizure, which induce cytotoxic edema, can manifest as hyperintense lesions on DWI. ${ }^{7}$ Together these observations indicate that even if a patient with transient amnesia shows multiple lesions on DWI, clinicians should be cautious to determine the possible etiology and therapeutic strategy.

\section{Conflicts of Interest}

We have no affiliations with or involvement in any organization or entity with any financial or nonfinancial interest in the reported study. The authors have no disclosures to report.

\section{REFERENCES}

1. Hodges JR, Warlow CP. Syndromes of transient amnesia: towards a classification. A study of 153 cases. J Neurol Neurosurg Psychiatry 1990;53:834-843.

2. Sedlaczek O, Hirsch JG, Grips E, Peters CN, Gass A, Wöhrle J, et al. Detection of delayed focal MR changes in the lateral hippocampus in transient global amnesia. Neurology 2004;62:2165-170.

3. Ay H, Furie KL, Yamada K, Koroshetz WJ. Diffusion-weighted MRI characterizes the ischemic lesion in transient global amnesia. Neurology 1998;51:901-903.

4. Saito K, Kimura K, Minematsu K, Shiraishi A, Nakajima M. Transient global amnesia associated with an acute infarction in the retrosplenium of the corpus callosum. J Neurol Sci 2003;210:95-97.

5. Valenstein E, Bowers D, Verfaellie M, Heilman KM, Day A, Watson RT. Retrosplenial amnesia. Brain 1987;110(Pt 6):1631-1646.

6. Sander K, Sander D. New insights into transient global amnesia: recent imaging and clinical findings. Lancet Neurol 2005;4:437-444.

7. Fisher M, Albers GW. Applications of diffusion-perfusion magnetic resonance imaging in acute ischemic stroke. Neurology 1999:52:1750-1756. 